\title{
A MOBILE GROUP DECISION MAKING MODEL FOR HETEROGENEOUS INFORMATION AND CHANGEABLE DECISION CONTEXTS
}

\author{
I. J. PEREZ \\ Department of Computer Science and Artificial Intelligence, University of Granada, Spain \\ ijperez@decsai.ugr.es \\ F. J. CABRERIZO \\ Department of Software Engineering and Computer Systems, \\ Distance Learning University of Spain (UNED), Spain \\ cabrerizo@issi.uned.es

\section{E. HERRERA-VIEDMA} \\ Department of Computer Science and Artificial Intelligence, University of Granada, Spain \\ viedma@decsai.ugr.es
}

Received 21 January 2011

Revised 5 July 2011

Accepted 10 September 2011

\begin{abstract}
The aim of this paper is to present a new mobile group decision making model to deal with heterogeneous information and changeable decision contexts. This model takes into account that experts have different backgrounds and knowledge levels, allowing to use different preference representations as fuzzy preference relations or linguistic preference relations with multigranular linguistic information. Furthermore, we allow to introduce some changes on the alternatives of the problem at every stage of the decision process. To do that: i) a mobile implementation is proposed to reduce the number of changes and ii) a mechanism to insert/remove alternatives is included in the model. Finally, our new decision model incorporates a feedback mechanism that sends recommendations to the experts in order to quickly obtain a high consensus level.
\end{abstract}

Keywords: Group decision making; consensus process; mobile devices; heterogeneous information; multigranular linguistic term sets; changeable contexts.

\section{Introduction}

Group decision making (GDM) is present in many real world situations. ${ }^{1-3}$ Consequently, the study and modelling of GDM problems is necessary and important not only in Decision Theory but also in areas such as Management Science, Operations Research, Politics, Social Psychology, and so on. In such circumstances, there are a set of alternatives to solve a problem and a group of experts that express their preferences by means of a set of evaluations and try to achieve a common solution. 
In an ideal GDM situation, all the experts could express their preferences in a precise way by using numerical values. Unfortunately, in many cases, due to the experts' background or the kind of information, experts can not represent their preferences precisely in a quantitative way. In these cases, it seems to be more adequate the use of qualitative concepts instead of numerical values. Several authors have provided interesting results on GDM with the help of fuzzy theory. ${ }^{4-10}$ Even, some of them have proposed the necessity of a linguistic approach to model that situations. ${ }^{11-18}$

A linguistic approach is an approximate technique which represents qualitative aspects as linguistic values by means of linguistic variables, that is, variables whose values are not numbers but words or sentences in a natural or artificial language. ${ }^{19,20}$ An important parameter to determine a linguistic approach is the number of linguistic variables, that is, the cardinality of the term set. There are cases where experts are non homogeneous in the sense of they have different background and levels of knowledge about the alternatives, and as consequence, they might use linguistic term sets with different granularity to express their preferences. In such cases, we say that GDM problem is defined in a multigranular fuzzy linguistic context. ${ }^{21-23}$ Given these facts, it seems reasonable that each expert chooses the most appropriate preference's representation to express his/her own opinions.

On the other hand, classical GDM models are frequently defined in fixed frameworks. In order to make the decision making process more realistic, it seems reasonable to design a model which is able to deal with changeable elements in decision making. The main element that could vary through the decision making process is the set of alternatives of the problem because they could depend on dynamical external factors, for example, the traffic, ${ }^{24}$ or the meteorological conditions. ${ }^{25}$ In such a way, we propose to solve changeable decision problems in which, at every stage of the process, the discussion is centered on a changeable set of alternatives.

Nowadays, we are realizing many significant advances in the way human interact with technology. The spread of e-services and wireless or mobile devices has increased accessibility to data and, in turn, influenced the way in which users make decisions while they are on the move. Users can make real-time decisions based on the most up-to-date data accessed via wireless devices, such as portable computers, mobile phones, and personal digital assistants (PDAs), which are usually carried all the time and allows to make decisions anytime and anywhere. Thus, the adoption of the latest mobile technologies extends opportunities and allows to carry out consensus processes where previously could not be correctly addressed. Such adoption is based on the assumption that if the communications are improved the decisions will be upgraded, because the discussion could be focussed on the problem with less time wasted on unimportant issues. ${ }^{26,27}$ In addition, as we have faster decisions, the number of changes in the context is drastically reduced.

The aim of this paper is to present a mobile GDM model to deal automatically with heterogeneous information and changeable GDM contexts. Hence, we propose the use of heterogeneous information, that is, we assume that the experts express their preferences by means of both fuzzy preference relations and multi-granular linguistic preference relations. The model also incorporates a mechanism to control the possible changes on the set of alternatives that could appear through the decision making process. As a result, we deal 
with heterogeneous scenarios at every stage of the decision process making uniform the different kinds of preferences in order to compute them and obtain a collective solution. Secondly, all possible changes of the alternatives of the decision context are managed. Thirdly, users receive recommendations to change their preferences in the best way to make a convergent process toward the consensus, reducing in this way the number of rounds to reach it. Finally, it is worth noting that the process is carried out by using mobile devices to send/receive information to/from the experts. Thus, due to the anytime and anywhere usage of this kind of devices, the communication flow is faster and consequently, the number of changes of the context is lower. Accordingly, we can conclude that with the use of this mobile GDM model, the user satisfaction and participation in the GDM process is improved.

To do so, the paper is set out as follows. Some considerations about classical, heterogeneous and changeable GDM framework are presented in Sec. 2. Section 3 deals with the new mobile model based on heterogeneous and changeable contexts. A case of use carried out with mobile devices is shown in Sec. 4. Finally, in Sec. 5 we point out our conclusions.

\section{Heterogeneous Information and Changeable Contexts in Group Decision Making Problems}

In this section, we show some information, motivations and background about classical, heterogeneous and changeable GDM frameworks.

\subsection{Group decision making problem}

A decision making process, consisting in deriving the best option from a feasible set, is present in just about every conceivable human task. It is obvious that the comparison of different actions according to their desirability in decision problems, in many cases, it cannot be done by using a single criterion or an unique person. Thus, we interpret the decision process in the framework of GDM. ${ }^{28,29}$

In a classical GDM situation there is a problem to solve, a solution set of possible alternatives, $X=\left\{x_{1}, x_{2}, \ldots, x_{n}\right\},(n \geq 2)$ and a group of two or more experts, $E=$ $\left\{e_{1}, e_{2}, \ldots, e_{m}\right\},(m \geq 2)$ characterized by their own ideas, attitudes, motivations and knowledge, who express their opinions about this set of alternatives to achieve a common solution. ${ }^{30-32}$

Usual resolution methods for GDM problems are composed by two different processes $^{6,17,33}$ (see Fig. 1):

(1) Consensus process: In any decision process, it is preferable that the experts reach a high degree of consensus on the solution set of alternatives. Thus, this process refers to how to obtain the maximum degree of consensus or agreement among the experts on the solution alternatives.

(2) Selection process: This process consists in how to obtain the solution set of alternatives from the opinions on the alternatives given by the experts. 


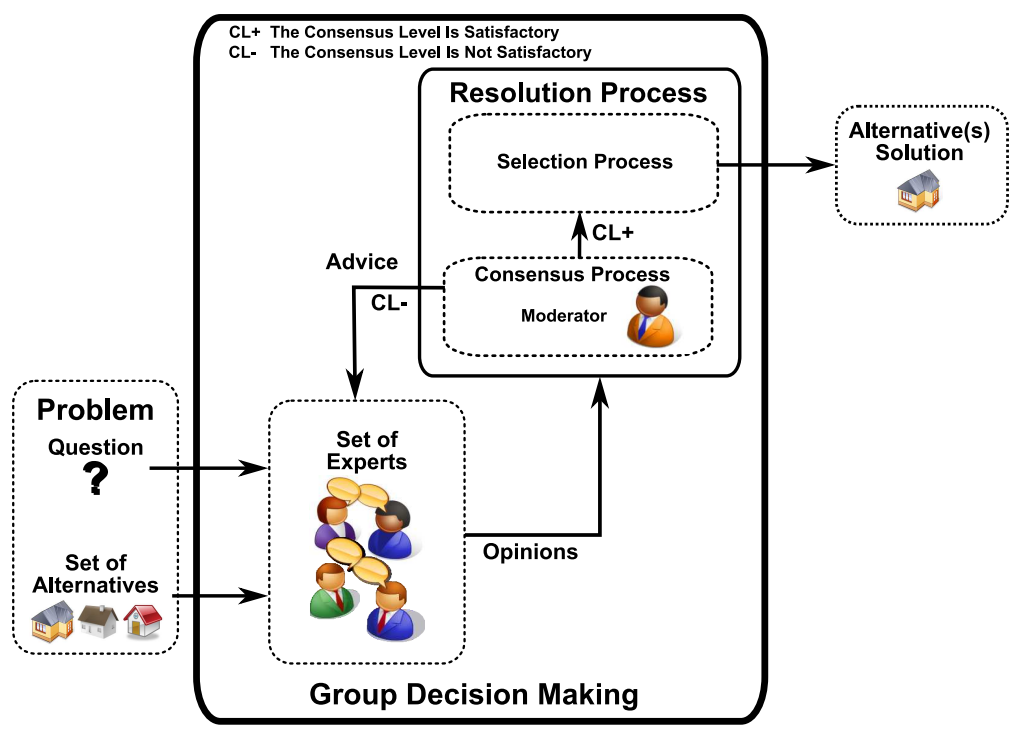

Fig. 1. Resolution process of a GDM.

\subsection{Heterogeneous information and changeable contexts in group decision making frameworks}

Recently, GDM problems have been studied and approached from different angles, showing that this kind of problems are not always under fixed conditions and there exist many different heterogeneous and changeable GDM contexts.

\subsubsection{Heterogeneous preferences' representation}

In some GDM situations an individual may have vague information about the preference degree of the alternative $x_{i}$ over $x_{j}$ and can not express his preference with an exact numerical value. Then, a more realistic approach is to use linguistic assessments instead of numerical values, that is, to suppose that the preferences are assessed by means of linguistic terms. ${ }^{19,34}$ Thus, the expert's knowledge degree on the problem determines the way and domain in which this expert is able to express his preferences. In such a way, those experts with high knowledge degree can give their opinions by using rigorous quantitative (numeric) variables and others experts with low knowledge degree could express their preferences in a non precise qualitative (linguistic) way. Therefore, the context must be defined using both quantitative and qualitative concepts. In this paper we assume that the experts could use two kind of preference relations to represent their preferences. ${ }^{35-41}$

\section{- Fuzzy preference relations}

Fuzzy preference relations (FPRs) are widely used in this kind of problems because they are more informative than preference orderings or utility functions, ${ }^{36}$ allowing the comparison of the alternatives in a pair by pair basis. Thus, users have much more freedom 
at giving their preferences and they can gain expressivity against other preference representations. By definition, a fuzzy preference relation $P^{h} \subset X \mathrm{x} X$, given by an expert $e_{h}$ is a fuzzy set defined on the product set $X \times X$, that is characterized by a membership function $\mu_{P^{h}}: X \mathrm{X} X \rightarrow[0,1]$, where $\mu_{P^{h}}\left(x_{i}, x_{j}\right)=p_{i j}^{h}$ denotes the preference degree of the expert $e_{h}$ on the alternative $x_{i}$ over $x_{j}$.

When cardinality of $X$ is small, the preference relation may be conveniently represented by an $n \times n$ matrix $P^{h}=\left(p_{i j}^{h}\right)$.

\section{- Multigranular fuzzy linguistic preference relations}

Fuzzy linguistic modelling is an approach based on the concept of linguistic variable to deal with qualitative assessments. In this approach, assessments of the preferences on pairs of alternatives are provided in the form of linguistic terms or labels of a linguistic term set $S=\left\{s_{0}, s_{1}, \ldots, s_{g}\right\}$. An important issue to analyze is the granularity or cardinality of the linguistic term set. The granularity of $S$ should be small enough so as not to impose useless precision levels on the users but large enough to allow a discrimination of the assessments in a limited number of degrees. In addition, the following properties are assumed:

(1) The set $S$ is ordered: $s_{i} \geq s_{j}$ if $i \geq j$.

(2) There is the negation operator: $N E G\left(s_{i}\right)=s_{j} \mid j=(g-i)$.

(3) There is the max operator: $M A X\left(s_{i}, s_{j}\right)=s_{i}$ if $s_{i} \geq s_{j}$.

(4) There is the min operator: $\operatorname{MIN}\left(s_{i}, s_{j}\right)=s_{i}$ if $s_{i} \leq s_{j}$.

The semantics of the linguistic terms can be given in three different ways: ${ }^{42}$ i) implicit numerical scale, ii) explicit quantitative or fuzzy scales and iii) operating directly on qualitative scales. In this paper, we assume the case which defines each linguistic term by using a fuzzy number on the $[0,1]$ interval. One way to characterize a fuzzy number is by using a representation based on parameters of its membership function. ${ }^{43,44}$ For example, the following semantics can be assigned to a set of seven terms:

- $N=$ Null $=(0,0,0.17)$

- VL $=$ VeryLow $=(0,0.17,0.33)$

- $L=$ Low $=(0.17,0.33,0.50)$

- $M=$ Medium $=(0.33,0.50,0.67)$

- $H=H i g h=(0.50,0.67,0.83)$

- $V H=$ VeryHigh $=(0.67,0.83,1)$

- $P=$ Perfect $=(0.83,1,1)$

The ideal situation for GDM problems defined in linguistic domains would be that all the experts use the same linguistic term set $S$ to express their preferences about the alternatives. However, in some cases, experts may belong, e.g., to distinct research areas, and therefore could have different background and levels of knowledge. A consequence of this is that they need to express their preferences by using linguistic term sets with different granularity $S_{h}=\left\{s_{0}^{h}, \ldots, s_{g_{h}}^{h}\right\}, h, \in\{1,2, \ldots, m\}$. In such a case, the GDM problem is defined in a multigranular fuzzy linguistic context. ${ }^{21,22}$ In our case, we propose the use of multigranular fuzzy linguistic preference relations to represent experts' preferences. The multigranular linguistic approach has proven its usefulness 
in many problems, e.g., in decision making, quality evaluation, information retrieval models, etc. ${ }^{45-52}$

A Multigranular Fuzzy Linguistic Preference Relation (MFLPR) $P^{h}$ given by an expert $e_{h}$ is a fuzzy set defined on the product set $X \times X$, that is characterized by a linguistic membership function $\mu_{P^{h}}: X \times X \longrightarrow S_{h}$, where the value $\mu_{P^{h}}\left(x_{i}, x_{j}\right)=$ $p_{i j}^{h}$ is interpreted as the linguistic preference degree of the alternative $x_{i}$ over $x_{j}$ for the expert $e_{h}$.

\subsubsection{Changeable contexts}

Frequently, the proposed resolution methods for GDM problems are static, that is, it is assumed that the elements of the problem (alternatives and experts acting in the problem) remain fixed throughout the decision making process. However, in real decision situations we find several GDM scenarios in which the number of alternatives could vary during the decision making process. ${ }^{38,53}$ Sometimes, when the decision process is slow or it takes a long time, the set of feasible alternatives has to be changeable because his availability or feasibility could change during the decision making time. For example, in e-commerce decision frameworks, where the alternatives are the items that could be bought, it is possible that the availability of some of these items changes while experts are discussing and making the decision, even, new good items might become available. Therefore, in this paper we assume GDM problems with a changeable set of alternatives.

\section{A New GDM Model Based on Heterogeneous Information and Changeable Decision Contexts}

In this section, we present a new GDM model that incorporates some mechanisms to manage the changes of the context that might happen during the decision process. Furthermore, the model is specifically designed to give freedom to the experts in the way that they provide their preferences with heterogeneous information, that is by means of both preference relations, fuzzy preference relations and multi-granular linguistic preference relations. Thus, the model has to adapt not only to the initial circumstances but also to the changes of the context. In such a way, changeable GDM processes with heterogeneous information could be developed and we can simulate with more accuracy level the real processes of human decision making which are carried out in changeable environments as the Web, commerce, financial investment, health, navigation, natural resources management and so on.

This new adaptive GDM model is composed of the following five processes (see Fig. 2):

(1) Format and domain management

(2) Consensus process

(3) Selection process

(4) Changeable context management

(5) Feedback process 


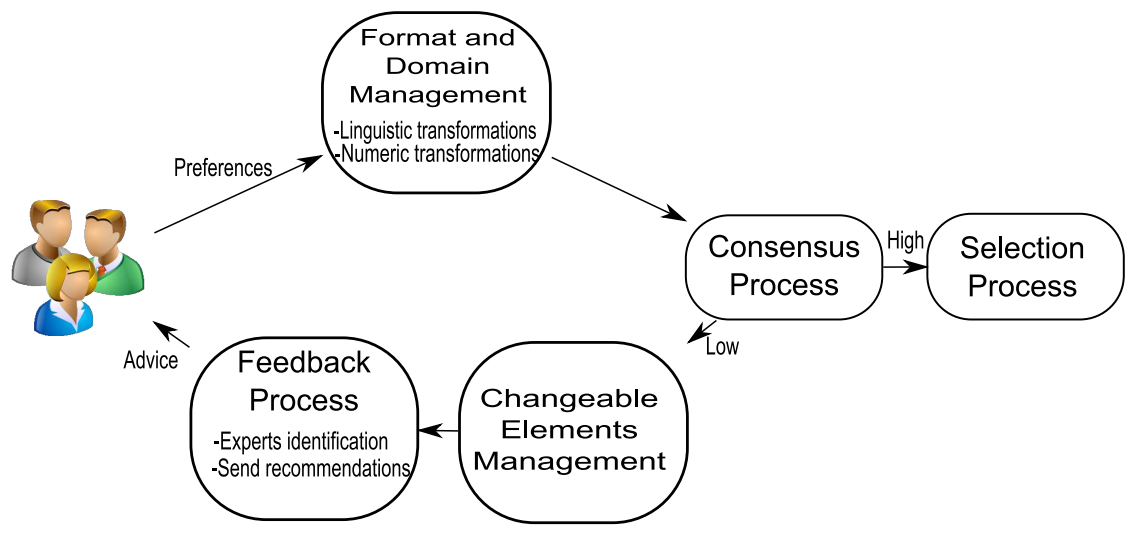

Fig. 2. Structure of the new GDM model for changeable contexts.

\subsection{Format and domain management}

Given that we assume two kind of preference relations, in our GDM model it is necessary to make the information uniform before applying the consensus and selection processes.

To make uniform multigranular fuzzy linguistic information, experts' preferences have to be transformed (using a transformation function) into a single domain or linguistic term set that we call the basic linguistic term set (BLTS), denoted by $S_{T} \cdot{ }^{21}$ To do this, it seems reasonable to impose a cardinality high enough to maintain the uncertainty degrees associated with each one of the possible domains to be unified. This means that the cardinality of the BLTS has to be as high as possible. Therefore, in a general multigranular fuzzy linguistic context, to select $S_{T}$, we proceed as it was proposed in Ref. 21.

(1) If there is only one linguistic term set, from the set of different domains to be unified, with maximum cardinality, then we choose that one as the BLTS, $S_{T}$.

(2) If there are two or more linguistic term sets with maximum cardinality, then the selection of $S_{T}$ will depend on the semantics associated with them.

(a) If all of them have the same semantics, i.e., the same fuzzy membership functions associated with the linguistic terms but with different syntax, then any one of them could be selected as $S_{T}$.

(b) If two or more of them have different semantics, then $S_{T}$ is defined as a generic linguistic term set with a number of terms greater than the number of terms a person is able to discriminate, which is normally 7 or $9 .{ }^{54}$

Once $S_{T}$ has been selected, the following multigranular transformation function is applied to transform every linguistic value into a fuzzy set defined on $S_{T}$. If $S=\left\{l_{0}, \ldots, l_{p}\right\}$ and $S_{T}=\left\{c_{0}, \ldots, c_{g}\right\}$ are two linguistic term sets, with $g \geq p$, then, a multigranular transformation function $\tau_{S, S_{t}}: S \rightarrow F\left(S_{T}\right)$ is defined as follows:

$$
\tau_{S, S_{t}}\left(l_{i}\right)=\left\{\left(c_{k}, \alpha^{k}\right) \mid \alpha^{k}=\max _{y} \min \left\{\mu_{l_{i}}(y), \mu_{c_{k}}(y)\right\}, k=\{0, \ldots, g\}\right\}
$$


Where $F\left(S_{T}\right)$ is the set of fuzzy sets defined on $S_{T}$, and $\mu_{l_{i}}(y)$ and $\mu_{c_{k}}(y)$ are the membership functions of the fuzzy sets associated with the linguistic terms $l_{i}$ and $c_{k}$, respectively.

In order to unify all the experts' preferences, different multigranular transformation functions $\tau_{S_{h}, S_{t}}$ are defined. Each linguistic preference value $p_{i j}^{h} \in S_{h}$ will be transformed in a fuzzy set $\tilde{p}_{i j}^{h}=\tau_{S_{h}, S_{t}}\left(p_{i j}^{h}\right)=\left\{\left(c_{k}, \alpha_{i j}^{k}\right) \mid k=\{0, \ldots, g\}\right\}$ on $S_{T}$. To simplify, we will use the membership degrees $\left(\alpha_{i j}^{0}, \ldots, \alpha_{i j}^{g}\right)$ to denote each fuzzy set $\tilde{p}_{i j}^{h}$

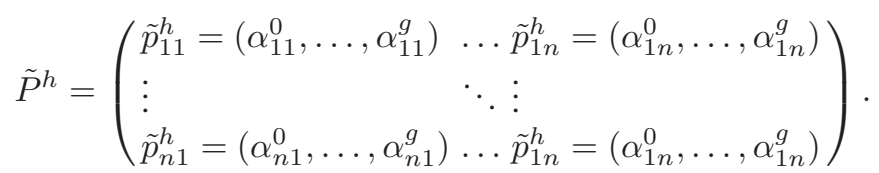

Finally, the system transforms each numeric FPR into a FLPR. To do so, we just have to compute its membership degree to each term of the BLTS.

\subsection{Consensus process}

Once the preferences have been given by the experts and the system has made this information uniform, we can compute the level of agreement achieved in the current round.

As experts might use linguistic term sets with different cardinality and semantics, each expert's linguistic preference $p_{i j}^{h}$ has been transformed in a fuzzy set $\tilde{p}_{i j}^{h}=\left(\alpha_{i j}^{h 0}, \ldots, \alpha_{i j}^{h g}\right)$. Some drawbacks related to the use of traditional distance measurements were pointed out in Ref. 22, and an alternative similarity function $s$ was proposed to overcome them. The similarity function takes as its arguments the central values of the fuzzy sets to compare. Given a fuzzy set $\tilde{p}_{i j}^{h}=\left(\alpha_{i j}^{h 0}, \ldots, \alpha_{i j}^{h g}\right)$, its central value defined as

$$
c v\left(\tilde{p}_{i j}^{h}\right)=\frac{\sum_{k=0}^{g} k \alpha_{i j}^{h k}}{\sum_{k=0}^{g} \alpha_{i j}^{h k}},
$$

represents the center of gravity of the information contained in the fuzzy set.

The similarity between two preference values, $s\left(\tilde{p}_{i j}^{h}, \tilde{p}_{i j}^{l}\right) \in[0,1]$, is defined as

$$
s\left(\tilde{p}_{i j}^{h}, \tilde{p}_{i j}^{l}\right)=1-\frac{\left|c v\left(\tilde{p}_{i j}^{h}\right)-c v\left(\tilde{p}_{i j}^{l}\right)\right|}{g} .
$$

Consensus degrees measure the agreement between experts' preferences. For each pair of experts $\left(e_{h}, e_{l}\right),(h<l)$, a similarity matrix $S M^{h l}$ is calculated with

$$
s m_{i j}^{h l}=s\left(\tilde{p}_{i j}^{h}, \tilde{p}_{i j}^{l}\right) \mid i, j=\{1, \ldots, n\} \wedge i \neq j .
$$

Then, a consensus matrix, $C M$, is calculated by aggregating all the similarity matrices using the arithmetic mean as the aggregation function $\phi$ :

$$
c m_{i j}=\phi\left(s m_{i j}^{12}, s m_{i j}^{13}, \ldots, s m_{i j}^{1 m}, s m_{i j}^{23}, \ldots, s m_{i j}^{(m-1) m}\right) .
$$

Once the similarity and consensus matrices are computed we proceed to obtain the consensus degrees at the three different levels to obtain a global consensus degree, called consensus on the relation: 
(1) Consensus degree on pairs of alternatives. The consensus degree on a pair of alternatives $\left(x_{i}, x_{j}\right)$, denoted $c p_{i j}$, is defined to measure the consensus degree amongst all the experts on that pair of alternatives:

$$
c p_{i j}=c m_{i j}
$$

(2) Consensus degree on alternatives. The consensus degree on alternative $x_{i}$, denoted $c a_{i}$, is defined to measure the consensus degree amongst all the experts on that alternative:

$$
c a_{i}=\frac{\sum_{j=1 ; j \neq i}^{n}\left(c p_{i j}+c p_{j i}\right)}{2(n-1)}
$$

(3) Consensus degree on the relation. The consensus degree on the relation, denoted $C R$, is defined to measure the global consensus degree amongst all the experts' opinions:

$$
C R=\frac{\sum_{i=1}^{n} c a_{i}}{n}
$$

When the consensus measure $C R$ has not reached the minimum required consensus level $C L$ and the number of rounds has not reached a maximum number of iterations (defined prior to the beginning of the decision process), the experts' opinions that are hindering the agreement must be modified. The value of $C L$ will obviously depend on the particular problem we are dealing with. When the consequences of the decision to be made are of utmost importance, the minimum level of consensus required to make that decision should be logically as high as possible. At the other extreme, when the decision's consequences are not really serious (but are still important), and it is urgent to obtain a solution of the problem, a lower $C L$ implies an small number of consensus rounds to reach the agreement, and consequently, faster decisions.

\subsection{Selection process}

In order to obtain a collective assessment from the whole group of experts, we apply a selection process which has two phases: ${ }^{3}$ (i) aggregation and (ii) exploitation.

\section{- Aggregation phase:}

This phase defines a collective preference relation, $P^{c}=\left(p_{i j}^{c}\right)$, obtained by means of the aggregation of all individual fuzzy preference relations $\left\{P^{1}, P^{2}, \ldots, P^{m}\right\}$. It indicates the global preference between every pair of alternatives according to the majority of experts' opinions. ${ }^{55,56}$ Clearly, because $p_{i j}^{h}=\left(\alpha_{i j}^{h 0}, \ldots, \alpha_{i j}^{h g}\right)$ then $p_{i j}^{c}=\left(\alpha_{i j}^{c 0}, \ldots, \alpha_{i j}^{c g}\right)$ with $\alpha_{i j}^{c k}=\phi\left(\alpha_{i j}^{1 k}, \ldots, \alpha_{i j}^{m k}\right)$, which means that $p_{i j}^{c}$ is also a fuzzy set defined on $S_{T}$. An useful aggregation operator is the Ordered Weighted Averaging (OWA) operator defined by Yager in Ref. 57. As we are handling a set of experts who come from different backgrounds, it seems reasonable that they present different importance degrees. To deal with this situation, before computing any aggregation, we might define a set of suitable weight values and choose a good aggregation operator ${ }^{58,59}$ which should take into account these weight values. To do that, we use the Induced OWA (IOWA) operator. ${ }^{60,61}$ 
- Exploitation phase:

This phase transforms the global information about the alternatives into a global ranking of them, from which the set of solution alternatives is obtained. The global ranking is obtained applying two choice degrees of alternatives to the collective fuzzy preference relation: ${ }^{62}$ the quantifier guided dominance degree (QGDD) and the quantifier guided non dominance degree (QGNDD).

(1) $Q G D D_{i}$ : This quantifier guided dominance degree quantifies the dominance that one alternative $x_{i}$ has over all the others in a fuzzy majority sense:

$$
Q G D D_{i}=\phi_{Q}\left(p_{i 1}^{c}, p_{i 2}^{c}, \ldots, p_{i(i-1)}^{c}, p_{i(i+1)}^{c}, \ldots, p_{i n}^{c}\right)
$$

This measure allows us to define the set of non-dominated alternatives with maximum linguistic dominance degree:

$$
X^{Q G D D}=\left\{x_{i} \in X \mid Q G D D_{i}=\sup _{x_{j} \in X} Q G D D_{j}\right\}
$$

(2) $Q G N D D_{i}$ : This quantifier guided non-dominance degree gives the degree in which each alternative $x_{i}$ is not dominated by a fuzzy majority of the remaining alternatives:

$$
\begin{aligned}
Q G N D D_{i}= & \phi_{Q}\left(N E G\left(p_{1 i}^{s}\right), N E G\left(p_{2 i}^{s}\right), \ldots, N E G\left(p_{(i-1) i}^{s}\right),\right. \\
& \left.N E G\left(p_{(i+1) i}^{s}\right), \ldots, N E G\left(p_{n i}^{s}\right)\right)
\end{aligned}
$$

where

$$
p_{i j}^{s}= \begin{cases}c_{0} & \text { if } p_{i j}^{c}<p_{j i}^{c} \\ p_{i j}^{c}-p_{j i}^{c} & \text { if } p_{i j}^{c} \geq p_{j i}^{c}\end{cases}
$$

represents the degree in which $x_{i}$ is strictly dominated by $x_{j}$. The set of of nondominated alternatives with maximum linguistic non-dominance degree is

$$
X^{Q G N D D}=\left\{x_{i} \in X \mid Q G N D D_{i}=\sup _{x_{j} \in X} Q G N D D_{j}\right\} .
$$

Finally, the solution $X_{\text {sol }}$ is obtained as:

$$
X_{\text {sol }}=X^{Q G D D} \cap X^{Q G N D D} .
$$

\subsection{Changeable elements management}

Probably, the most useful contribution of this paper is the new process to manage changeable elements. In many real world decision contexts as health, commerce, and so on, due to different factors, some elements of the problem could vary throughout the decision process.

In order to make the decision making process more realistic, we provide a new tool to deal with appropriate changes of the set of alternatives of the problem. This tool is defined by a method which allows the revision of the alternatives, by removing old items or inserting new ones. To do so, the method identifies those unavailable or unfeasible alternatives and remove them if necessary. Then, if new good alternatives have appeared at a time, they can be inserted as elements of the problem and be considered in the next consensus round. 
It is worth noting that we assume that alternatives are independent and the inclusion or elimination of one of them can not change the features of the remaining ones.

Thus, the method has two different phases: (1) Remove old bad elements and (2) Insert new good elements.

(1) The first phase identifies alternatives whose initial feasibility or availability have been reduced during the decision process. To do that, the tool checks the availability and the QGDD degree of each alternative. If any of them is not available or it has a low QGDD, this alternative is a candidate to be removed.

(2) The second phase manages the opposite situation, that is, when some new elements have emerged. Basically, the system checks if some new good experts or alternatives have appeared in the decision context due to some dynamic external factors. If this is the case, these alternatives are candidates to be considered as elements of the problem.

Finally, experts are informed about the candidates to be removed and inserted and they have to express their agree or disagree with each of the changes. Then, the tool acts on each particular change possibility according to the majority of the experts opinions.

In most of the cases, changes happen because the GDM process depends not only on the server operation, but also on the experts interaction. Unfortunately, experts are not always online, consequently, the problem resolution can be delayed while experts send their preferences. This wasted time could be avoided by running the system on mobile devices, that can be used at anytime and anywhere, reaching faster decisions and avoiding the necessity of management of many of the changes produced in the context if experts were late sending their opinions.

\subsection{Feedback process}

To guide the change of the experts' opinions, the model simulates a group discussion session in which a feedback mechanism is applied to quickly obtain a high consensus level. This mechanism is able to substitute the moderator's actions in the consensus reaching process. The main problem for the feedback mechanism is how to find a way of making individual positions converge and, therefore, how to support the experts in obtaining and agreeing with a particular solution. To do that, we compute others consensus measures, called proximity measures. . $^{6} 17$

These measures evaluate the agreement between the individual experts' opinions and the group opinion. To compute them for each expert, we need to use the collective fuzzy linguistic preference relation, $P^{c}=\left(p_{i j}^{c}\right)$, previously defined as the aggregation of the individual preference relations.

Clearly, the similarity functions defined in Ref. 22 can be used to evaluate the agreement between each individual expert's preferences, $P^{h}$, and the collective preferences, $P^{c}$. Therefore, the measurement of proximity is carried out in two steps.

(1) For each expert, $e_{h}$, a proximity matrix, $P M^{h}=\left(p m_{i j}^{h}\right)$, is obtained where

$$
p m_{i j}^{h}=s\left(p_{i j}^{h}, p_{i j}^{c}\right) \text {. }
$$


(2) Computation of proximity measures at three different levels:

(a) Proximity measure on pairs of alternatives, $p p_{i j}^{h}$. It measures the proximity between the preferences on each pair of alternatives of the expert $e_{h}$ and the group.

$$
p p_{i j}^{h}=p m_{i j}^{h} .
$$

(b) Proximity measure on alternatives, $p a_{i}^{h}$. It measures the proximity between the preferences on each alternative $x_{i}$ of the expert $e_{h}$ and the group.

$$
p a_{i}^{h}=\frac{\sum_{j=1, j \neq i}^{n} p p_{i j}^{h}}{(n-1)} .
$$

(c) Proximity measure on the relation, $p r^{h}$. It measures the global proximity between the preferences of each expert $e_{h}$ and the group.

$$
p r^{h}=\frac{\sum_{i=1}^{n} p a_{i}^{h}}{n} .
$$

These measures let us to build a feedback mechanism so that experts change their opinions and narrow their positions. To do so, the production of advice to achieve a solution with the highest possible degree of consensus is carried out in two phases: Identification phase and Recommendation phase.

(1) Identification phase. We must identify the experts, alternatives and pairs of alternatives that are contributing less to reach a high degree of consensus.

(a) Identification of experts. We identify the set of experts, $E X P C H$, that should receive advice on how to change some of their preference values:

$$
E X P C H=\left\{h \mid p r^{h}<\gamma\right\} .
$$

Where $\gamma$ is the minimum proximity level required for the expert to be noted to change.

(b) Identification of alternatives. We identify the alternatives whose associated assessments should be taken into account by the above experts in the change process of their preferences;

$$
A L T_{h}=\left\{x_{i} \in X \mid p a_{i}^{h}<\gamma \wedge h \in E X P C H\right\} .
$$

(c) Identification of pairs of alternatives. In this step we identify the particular pairs of alternatives $\left(x_{i}, x_{j}\right)$ whose respective assessments $p_{i j}^{h}$ the expert $e_{h}$ should change.

$$
P A L T_{h}=\left\{\left(x_{i}, x_{j}\right) \mid p p_{i j}^{h}<\gamma \wedge x_{i} \in A L T_{h} \wedge h \in E X P C H\right\} .
$$

(2) Recommendation phase. In this phase we recommend expert changes of their preferences according to two kinds of rules:

(a) Rules to change the opinions. We must find out the direction of change to be applied to the preference assessment $p_{i j}^{h}$, with $\left(x_{i}, x_{j}\right) \in P A L T_{h}$. To do this, we define the following two direction rules. It is worth to note that if one of the alternatives of the pair $\left(x_{i}, x_{j}\right)$ has been replaced in the managing process 
of dynamic information, that pair has to be removed from the set $P A L T_{h}$, as there is not need to provide rules for alternatives that have been removed from the alternatives' set.

- If $p_{i j}^{h}>p_{i j}^{c}$, the expert $e_{h}$ should decrease the assessment associated to the pair of alternatives $\left(x_{i}, x_{j}\right)$.

- If $p_{i j}^{h}<p_{i j}^{c}$, the expert $e_{h}$ should increase the assessment associated to the pair of alternatives $\left(x_{i}, x_{j}\right)$.

\section{Example}

In this section, we will illustrate a simple usage scenario of the system to check how the model works. We should point out that the system could deal with complex problems perfectly, because the prototype allows changeable sets of alternatives, it manages their inputs and outputs in real time, and it can also address problems with large sets of alternatives. When all alternatives cannot be displayed on a mobile screen at the same time, the remaining ones can be ordered in a supply list and be evaluated later in the process. Therefore, the system can support a big number of alternatives to solve complex problems.

Let us suppose that the board of directors of a company, composed of 4 members (experts), would like to buy a new powerful computer in order to update their software to the new technologies. Consequently, they have to decide the most appropriate one to cover the requirements of the new system. Thus, they consider four alternatives $X=\{$ Acer, Sony, Toshiba, Apple $\}$ and it is necessary to reach consensus to choose one of them.

In this initial context, we have to take into account that the directors' background is heterogeneous, so, they can not express their preferences in the same way. Moreover, we need to use a GDM model that manages changeable frameworks because in commerce decision situations, the items can change their availability quite often. In particular, our mobile GDM model was used because it has been designed to deal with heterogeneous information and this kind of changes in the context of the decision problem.

\subsection{First round}

Expert $e_{1}$ started giving his opinions by using a FPR, $e_{2}$ by using a MFLPR with the label set $\mathrm{A}$, and $e_{3}$ by using a MFLPR with the label set B (see Fig. 3):

- Label set A:

$$
\begin{aligned}
& -a_{0}=(0,0,0.13) \\
& -a_{1}=(0,0.13,0.25) \\
& -a_{2}=(0.13,0.25,0.38) \\
& -a_{3}=(0.25,0.38,0.5) \\
& -a_{4}=(0.38,0.5,0.63) \\
& -a_{5}=(0.5,0.63,0.75) \\
& -a_{6}=(0.63,0.75,0.88)
\end{aligned}
$$




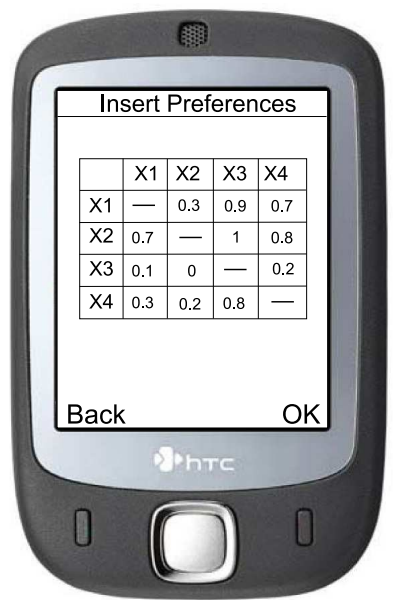

(a) Expert 1

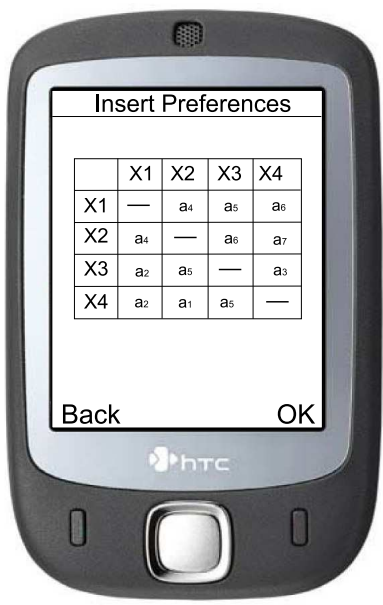

(b) Expert 2

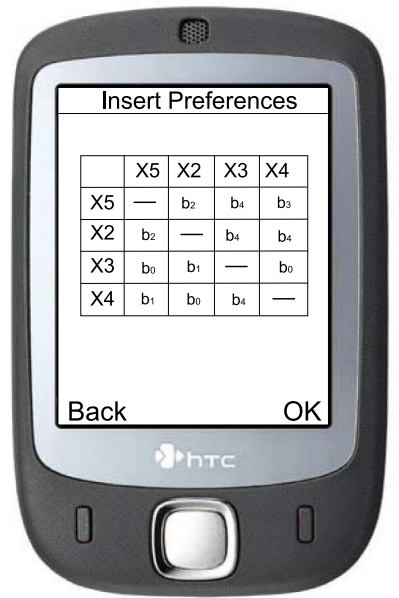

(c) Expert 3

Fig. 3. Expert's preferences.

$$
\begin{aligned}
& -a_{7}=(0.75,0.88,1) \\
& -a_{8}=(0.88,1,1)
\end{aligned}
$$

- Label set B:

$$
\begin{aligned}
& -b_{0}=(0,0,0.25) \\
& -b_{1}=(0,0.25,0.5) \\
& -b_{2}=(0.25,0.5,0.75) \\
& -b_{3}=(0.5,0.75,1) \\
& -b_{4}=(0.75,1,1)
\end{aligned}
$$

\subsubsection{Format and domain management: making the information uniform}

According to the conditions in Sec. 3.1, $S_{T}=A$. We unify the different linguistic term sets by using the transformation functions $\left\{\tau_{A, S_{t}}, \tau_{B, S_{t}}\right\}$, then we compute the membership degrees to each term of $S_{T}$ of the preferences $p_{i j}^{1}$.

$$
\begin{aligned}
& \tau_{A, S_{t}}: \\
& a_{0} \rightarrow(1,0,0,0,0,0,0,0,0) \\
& a_{1} \rightarrow(0,1,0,0,0,0,0,0,0) \\
& a_{2} \rightarrow(0,0,1,0,0,0,0,0,0) \\
& a_{3} \rightarrow(0,0,0,1,0,0,0,0,0) \\
& a_{4} \rightarrow(0,0,0,0,1,0,0,0,0) \\
& a_{5} \rightarrow(0,0,0,0,0,1,0,0,0) \\
& a_{6} \rightarrow(0,0,0,0,0,0,1,0,0) \\
& a_{7} \rightarrow(0,0,0,0,0,0,0,1,0) \\
& a_{8} \rightarrow(0,0,0,0,0,0,0,0,1)
\end{aligned}
$$




\subsubsection{Consensus process}

Computing consensus degree: The consensus degree is obtained at the three different levels. First, the similarity matrix for each pair of experts is computed, and so the consensus matrix is obtained. Then, the consensus degrees on pairs of alternatives, alternatives, and global relation are obtained from the consensus matrix.

(1) Consensus Matrix:

$$
C M=\left(\begin{array}{cccc}
- & 0.66 & 0.50 & 0.38 \\
0.66 & - & 0.42 & 0.38 \\
0.36 & 0.59 & - & 0.66 \\
0.36 & 0.52 & 0.66 & -
\end{array}\right)
$$

(2) Consensus on pairs of alternatives: The element $(i, j)$ of $C M$ represents the consensus degrees on the pair of alternatives $\left(x_{i}, x_{j}\right)$, thus, $c p_{i j}=c m_{i j}$.

(3) Consensus on alternatives:

$$
c a_{1}=0.49 \quad c a_{2}=0.54 \quad c a_{3}=0.53 \quad c a_{4}=0.49
$$

(4) Consensus on the relation:

$$
C R=0.51
$$

Controlling the consensus process: In this step of the consensus model, the global consensus value, $C R$, is compared with the minimum consensus threshold, $C L$. In this example, we have decided to use the value, $C L=0.75$. As $C R<C L$ and number of rounds $<$ MaxRounds, it is concluded that the consensus among the experts is not high enough.

\subsubsection{Changeable elements management}

As soon as the system has verified that the minimum consensus level among the experts has not been reached and before beginning a new round of consensus, it is necessary to update all the information of the problem that there could be changed during the process.

In this case, the alternatives have changed. The alternative $x_{1}$ provided by "Acer" is not available because it is out of stock. Moreover, a new good alternative provided by "Dell" has recently appeared in the computers market. Therefore, the model asks the experts if they agree with the changes and as the answer is affirmative, Dell is now the new alternative $x_{1}$. The experts will be informed about it and then they are urged to refill their preferences about the new alternative.

\subsubsection{Feedback mechanism}

- Computation of proximity measures:

(1) Proximity matrices:

$$
P M^{1}=\left(\begin{array}{cccc}
- & 0.75 & 0.75 & 0.50 \\
0.75 & - & 0.75 & 0.50 \\
0.50 & 1.00 & - & 0.00 \\
0.50 & 0.75 & 0.75 & -
\end{array}\right)
$$




$$
\begin{aligned}
P M^{2} & =\left(\begin{array}{cccc}
- & 1.00 & 0.50 & 0.75 \\
1.00 & - & 0.75 & 0.75 \\
0.75 & 0.75 & - & 1.00 \\
0.75 & 0.75 & 1.00 & -
\end{array}\right) \\
P M^{3} & =\left(\begin{array}{cccc}
- & 0.75 & 0.75 & 0.75 \\
0.75 & - & 0.25 & 0.50 \\
0.25 & 0.50 & - & 0.75 \\
0.50 & 0.75 & 0.75 & -
\end{array}\right)
\end{aligned}
$$

(2) Proximity on pairs of alternatives: $P P^{h}=P M^{h}$.

(3) Proximity on alternatives (See Table 1):

Table 1. Proximity measures on alternatives.

\begin{tabular}{|c|c|c|c|}
\hline$x_{1}$ & $x_{2}$ & $x_{3}$ & $x_{4}$ \\
\hline$p a_{1}^{1}=0.66$ & $p a_{2}^{1}=0.66$ & $p a_{3}^{1}=0.50$ & $p a_{4}^{1}=0.66$ \\
\hline$p a_{1}^{2}=0.75$ & $p a_{2}^{2}=0.83$ & $p a_{3}^{2}=0.83$ & $p a_{4}^{2}=0.83$ \\
\hline$p a_{1}^{3}=0.75$ & $p a_{2}^{3}=0.50$ & $p a_{3}^{3}=0.50$ & $p a_{4}^{3}=0.66$ \\
\hline
\end{tabular}

(4) Proximity on the relation:

$$
p r^{1}=0.59 p r^{2}=0.81 p r^{3}=0.80
$$

- Production of advice:

(1) Identification phase:

(a) Identification of experts:

$$
\text { EXPCH }=\left\{e_{h} \mid p r^{h}<\operatorname{minProxDegree}=0.65\right\}=\left\{e_{1}\right\}
$$

(b) Identification of alternatives:

$$
\begin{aligned}
A L T_{1} & =\left\{x_{i} \in X \mid p a_{i}^{h}<\right.\text { minProxDegree } \\
& \left.=0.65 \wedge e_{h} \in E X P C H\right\}=\left\{x_{3}\right\}
\end{aligned}
$$

(c) Identification of pairs of alternatives to generate recommendations:

$$
P A L T_{1}=\left\{\left(x_{3}, x_{1}\right),\left(x_{3}, x_{2}\right),\left(x_{3}, x_{4}\right)\right\}
$$

(2) Recommendation phase:

In this phase, we have to take into account that alternative $x_{1}$ has been replaced in the previous process by a new one. So, $x_{1}$ does not need rules to be modified and there is a new alternative in the discussion set that needs new preference values (see Fig. 4). 


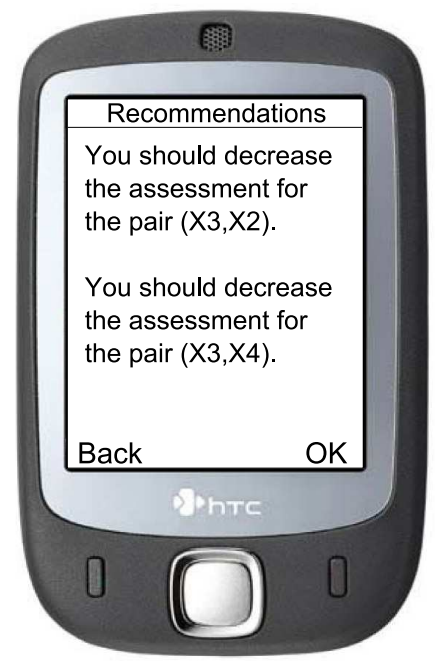

Fig. 4. Expert 1 recommendations.

(a) Rules to change the opinions:

- Because $x_{1}$ has been replaced, $p_{31}^{1}$ and $p_{13}^{1}$ do not need be modified.

- Because $p_{32}^{1}>p_{32}^{c}$, expert $e_{1}$ is advised to decrease the assessment of the alternative $x_{3}$ in respect of the alternative $x_{2}$.

- Because $p_{34}^{1}<p_{34}^{c}$, expert $e_{1}$ is advised to increase the assessment of the alternative $x_{3}$ in respect of the alternative $x_{4}$.

\subsection{Second round}

The experts send their preferences about the new discussion set of alternatives to start the second round of the consensus reaching process (see Fig. 5).

\subsubsection{Make information uniform and consensus process}

To make the information uniform, the system acts in the same way that in the previous round. Then, the consensus measure is computed.

Consensus on the relation: $C R=0.78$

Because $C R>C L$, then it is concluded that there is the required consensus amongst the experts, and consequently, the final solution should be obtained by applying the selection process.

\subsubsection{Selection process}

(1) Aggregation: The collective preference relation is computed by using the OWA operator. 


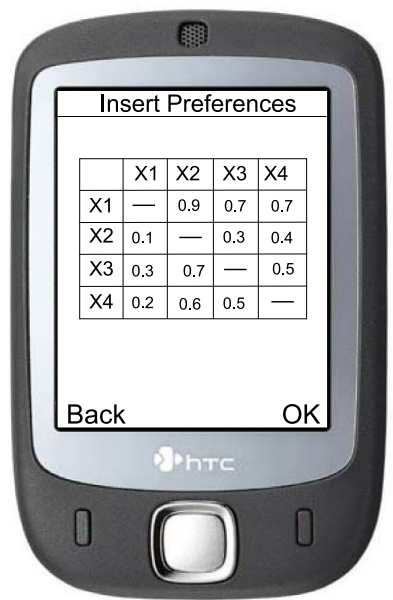

(a) Expert 1

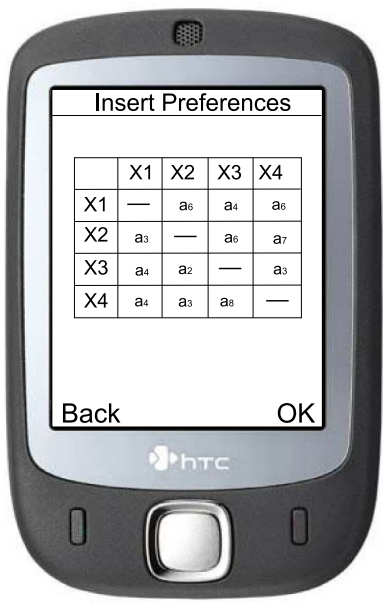

(b) Expert 2

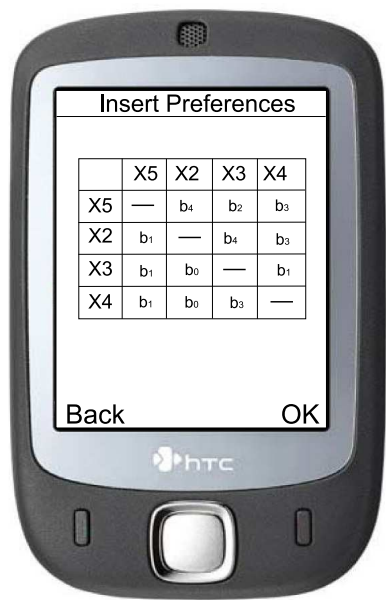

(c) Expert 3

Fig. 5. Experts' preferences.

Table 2. Choice degrees.

\begin{tabular}{|c|c|c|c|c|}
\hline & $x_{1}$ & $x_{2}$ & $x_{3}$ & $x_{4}$ \\
\hline$Q G D D_{i}$ & $\mathrm{VH}$ & $\mathrm{H}$ & $\mathrm{M}$ & $\mathrm{H}$ \\
\hline$Q G N D D_{i}$ & $\mathrm{P}$ & $\mathrm{VH}$ & $\mathrm{VH}$ & $\mathrm{VH}$ \\
\hline
\end{tabular}

(2) Exploitation: Using the linguistic quantifier "most of", we obtain the choice degrees (see Table 2).

Clearly, the maximal sets are:

$$
X^{Q G D D}=\left\{x_{1}\right\} \text { and } X^{Q G N D D}=\left\{x_{1}\right\}
$$

Finally, we can conclude that according to the experts' opinion, the computer provided by "DELL" offers the biggest price-quality ratio to meet their requirements.

\section{Conclusions}

We have presented a new mobile GDM model for changeable decision environments which allows experts to send/receive information at anytime and anywhere and also choose the best way to express their preferences.

In short, we can conclude that with this mobile GDM model we shall be able to model a wide range of GDM problems in which experts can interact in a really flexible way by using FPRs or MFLPRs to express their preferences and, if it is necessary, the alternatives of the problem could be modified during the decision process. 


\section{Acknowledgements}

This paper has been developed with the financing of FEDER funds in FUZZYLING Project TIN2007-61079, FUZZYLING-II Project TIN2010-17876, PETRI Project PET20070460, Andalusian Excellence Projects TIC-05299 and TIC-5991, and project of Ministry of Public Works 90/07.

\section{References}

1. E. Herrera-Viedma, J. García-Lapresta, J. Kacprzyk, H. Nurmi, M. Fedrizzi and S. Zadrozny, Consensual Processes (Springer-Verlag, Berlin, 2011).

2. J. Kacprzyk and M. Fedrizzi, Multiperson Decision Making Models using Fuzzy Sets and Possibility Theory (Kluwer Academic Publishers, Dordrecht, 1990).

3. M. Roubens, Fuzzy Sets and Systems 90, 199 (1997).

4. S. Alonso, E. Herrera-Viedma, F. Chiclana and F. Herrera, Int. J. Information Technology \& Decision Making 8, 313 (2009).

5. F. E. Boran, S. Genç, M. Kurt and D. Akay, Expert Systems with Applications 36, 11363 (2009).

6. F. Cabrerizo, J. Moreno, I. Perez and E. Herrera-Viedma, Soft Computing 14, 451 (2010).

7. F. Chiclana, F. M. L. Martínez, E. Herrera-Viedma and S. Alonso, Int. J. Uncertainty, Fuzziness and Knowledge-Based Systems 16, 35 (2008).

8. J. Fodors and M. Roubens, Fuzzy Preference Modelling and Multicriteria Decision Support (Kluwer Academic Publishers, Dordrecht, 1994).

9. F. Herrera, E. Herrera-Viedma and J. Verdegay, Information Sciences 85, 223 (1995).

10. A. Sanayei, S. F. Mousavi and A. Yazdankhah, Expert Systems with Applications 37, 24 (2010).

11. S. Alonso, F. Cabrerizo, F. Chiclana, F. Herrera and E. Herrera-Viedma, Int. J. Intelligent Systems 24, 201 (2009).

12. S. Alonso, E. Herrera-Viedma, F. Chiclana and F. Herrera, Information Sciences 180, 4477 (2010).

13. D. Ben-Arieh and Z. Chen, IEEE Trans. Systems, Man, and Cybernetics. Part A: Systems and Humans 36, 558 (2006).

14. F. Cabrerizo, S. Alonso and E. Herrera-Viedma, Int. J. Information Technology \& Decision Making 8, 109 (2009).

15. F. Cabrerizo, I. Perez and E. Herrera-Viedma, Knowledge-Based Systems 23, 169 (2010).

16. F. Cabrerizo, J. Lǿpez-Gijǿn, A. Ruíz-Rodríguez and E. Herrera-Viedma, Int. J. Information Technology and Decision Making 9, 455 (2010).

17. F. Herrera, E. Herrera-Viedma and J. Verdegay, Fuzzy Sets and Systems 78, 73 (1996).

18. Z. Zhang and X. Chu, Expert Systems with Applications 36, 9150 (2009).

19. F. Herrera and E. Herrera-Viedma, Fuzzy Set and Systems 115, 67 (2000).

20. J. Kacprzyk and S. Zadrozny, Int. J. Uncertainty, Fuzziness and Knowledge-Based Systems $\mathbf{9}$, 89 (2001).

21. F. Herrera, E. Herrera-Viedma and L. Martinez, Fuzzy Set and Systems 114, 43 (2000).

22. E. Herrera-Viedma, L. Martinez, F. Mata and F. Chiclana, IEEE Trans. Fuzzy Systems 13, 644 (2005).

23. F. Mata, L. Martínez and E. Herrera-Viedma, IEEE Trans. Fuzzy Systems 17, 279 (2009).

24. H. Dia, Transportation Research Part C 10, 331 (2002).

25. H. Clarke, The Australian Journal of Agricultural and Resource Economics 52, 487 (2008).

26. J. Katz, Handbook of Mobile Communication Studies (The MIT Press, 2008).

27. J. Schiller, Mobile Communications, 2nd edn. (Addison Wesley, 2003).

28. F. Herrera, E. Herrera-Viedma and J. Verdegay, Int. J. Approximate Reasoning 16, 309 (1997). 
29. E. Herrera-Viedma, S. Alonso, F. Chiclana and F. Herrera, IEEE Trans. Fuzzy Systems 15, 863 (2007).

30. J. Lu, G. Zhang and D. Ruan, Soft Computing 12, 289 (2008).

31. J. Montero, Soft Computing 12, 177 (2008).

32. H. Nurmi, Soft Computing 12, 281 (2008).

33. F. Herrera, E. Herrera-Viedma and J. Verdegay, Fuzzy Sets and Systems 88, 31 (1997).

34. F. Herrera, S. Alonso, F. Chiclana and E. Herrera-Viedma, Fuzzy Optimization and Decision Making 8, 337 (2009).

35. G. Buyukozkan, O. Feyzioglu and D. Ruan, Computers in Industry 58, 392 (2007).

36. F. Chiclana, F. Herrera and E. Herrera-Viedma, Fuzzy Sets and Systems 97, 33 (1998).

37. F. Chiclana, F. Herrera and E. Herrera-Viedma, Fuzzy Sets and Systems 122, 277 (2001).

38. I. Perez, F. Cabrerizo and E. Herrera-Viedma, IEEE Trans. Systems, Man and Cybernetics. Part A: Systems and Humans 40, 1244 (2010).

39. E. Herrera-Viedma, F. Herrera and F. Chiclana, IEEE Trans. Systems, Man, and Cybernetics. Part A: Systems and Humans 32, 394 (2002).

40. Z. Xu, IEEE Trans. Systems, Man and Cybernetics. Part B: Cybernetics 37, 1500 (2007).

41. Z. Xu and J. Chen, IEEE Transactions on Systems, Man and Cybernetics. Part B: Cybernetics 38, 1356 (2008).

42. V. Torra, Int. J. Intelligent Systems 16, 513 (2001).

43. P. Bonissone and K. Decker, Uncertainty in Artificial Intelligence, 217 (1986).

44. J. Lawry and H. He, Int. J. Uncertainty, Fuzziness and Knowledge-Based Systems 16, 69 (2008).

45. J. García-Lapresta and L. Meneses, Fuzzy Sets and Systems 160, 3211 (2009).

46. E. Herrera-Viedma, O. Cordon, M. Luque, A. Lopez-Herrera and A. Muñoz, Int. J. Approximate Reasoning 34, 221 (2003).

47. E. Herrera-Viedma, G. Pasi, A. G. López-Herrera and C. Porcel, J. Am. Soc. Information Science and Technology 57, 538 (2006).

48. E. Herrera-Viedma and E. Peis, Information Processing \& Management 39, 195 (2003).

49. E. Herrera-Viedma, E. Peis, J. Morales, S. Alonso and K. Anaya, Int. J. Approximate Reasoning 46, 226 (2007).

50. C. Porcel, A. Lopez-Herrera and E. Herrera-Viedma, Expert Systems with Applications 36, 15173 (2009).

51. C. Porcel, J. Moreno and E. Herrera-Viedma, Expert Systems with Applications 36, 12520 (2009).

52. C. Porcel, J. del Castillo, M. Cobo, A. Ruiz-Rodriguez and E. Herrera-Viedma, Control and Cybernetics 39, 899 (2010).

53. I. Perez, F. Cabrerizo and E. Herrera-Viedma, Expert Systems with Applications 38, 1675 (2011).

54. G. Miller, Psychol. Rev. 63, 81 (1956).

55. Z. Xu, Int. J. Uncertainty, Fuzziness and Knowledge-Based Systems 17, 15 (2009).

56. J. Merigo, M. Casanovas and L. Martinez, Int. J. Uncertainty, Fuzziness and Knowledge-Based Systems 18, 287 (2010).

57. R. Yager, IEEE Trans. Systems, Man, and Cybernetics 18, 183 (1988).

58. V. Torra and Y. Narukawa, IEEE Trans. Fuzzy Systems 15, 1063 (2007).

59. V. Torra and Y. Narukawa, Modeling Decisions: Information Fusion and Aggregation Operators (Springer, 2007).

60. F. Chiclana, E. Herrera-Viedma, F. Herrera and S. Alonso, European J. Operational Research 182, 383 (2007).

61. R. Yager and D. Filev, IEEE Trans. on Systems, Man, and Cybernetics 29, 141 (1999).

62. F. Herrera and E. Herrera-Viedma, European J. Operational Research 120, 144 (2000). 